\title{
Upper Endoscopy up to 3 Years Prior to a Diagnosis of Gastric Cancer Is Associated With Lower Stage of Disease in a USA Multiethnic Urban Population, a Retrospective
} Study

\author{
Shailja C. Shah ${ }^{1,2}$, Chiaki Nakata ${ }^{3}$, Alexandros D. Polydorides ${ }^{1,4}$, Richard M. Peek Jr², Steven H. Itzkowitz \\ ${ }^{1}$ The Dr. Henry D. Janowitz Division of Gastroenterology, Icahn School of Medicine at Mount Sinai, New York, NY, USA; ${ }^{2}$ Division of Gastroenterology, \\ Hepatology, and Nutrition, Vanderbilt University Medical Center, Nashville, TN, USA; ${ }^{3}$ Icahn School of Medicine at Mount Sinai, New York, NY, USA; \\ ${ }^{4}$ Department of Pathology, Icahn School of Medicine at Mount Sinai, New York, NY, USA
}

Objectives: In the USA, certain races and ethnicities have a disproportionately higher gastric cancer burden. Selective screening might allow for earlier detection and curative resection. Among a USA-based multiracial and ethnic cohort diagnosed with non-cardia gastric cancer (NCGC), we aimed to identify factors associated with curable stage disease at diagnosis.

Methods: We retrospectively identified endoscopically diagnosed and histologically confirmed cases of NCGC at Mount Sinai Hospital in New York City. Demographic, clinical, endoscopic and histologic factors, as well as grade/stage of NCGC at diagnosis were documented. The primary outcome was the frequency of curable-stage NCGC (stage 0-1a) at diagnosis in patients with versus without an endoscopy negative for malignancy prior to their index exam diagnosing NCGC. Additional factors associated with curable-stage disease at diagnosis were determined.

Results: A total of 103 racially and ethnically diverse patients were included. Nearly $38 \%$ of NCGC were stage 0 -la, $34 \%$ stage Ib-III, and $20.3 \%$ stage IV at diagnosis. A significantly higher frequency of NCGC was diagnosed in curable stages among patients who had undergone an endoscopy that was negative for malignancy prior to their index endoscopy that diagnosed NCGC, compared to patients without a negative endoscopy prior to their index exam $(69.6 \%$ vs. $28.6 \%, p=0.003)$. A prior negative endoscopy was associated with $94.0 \%$ higher likelihood of diagnosing curable-stage NCGC $(p=0.003)$. No other factors analyzed were associated with curablestage NCGC at diagnosis.

Conclusions: Endoscopic screening and surveillance in select high-risk populations might increase diagnoses of curable-stage NCGC. These findings warrant confirmation in larger, prospective studies.

Key words: Early diagnosis, Gastrointestinal neoplasm, Helicobacter pylori, Intestinal metaplasia, Atrophic gastritis, Race factors

Received: November 14, 2018 Accepted: April 24, 2019

Corresponding author: Shailja C. Shah, MD, MPH

Division of Gastroenterology, Hepatology, and Nutrition, Vanderbilt University Medical Center, 2215 Garland Avenue, Nashville,

TN 37203, USA

E-mail: shailja.c.shah@vumc.org

This is an Open Access article distributed under the terms of the Creative Commons Attribution Non-Commercial License (http://creativecommons.org/licenses/by$\mathrm{nc} / 4.0 /$ ) which permits unrestricted non-commercial use, distribution, and reproduction in any medium, provided the original work is properly cited.

\section{INTRODUCTION}

Gastric cancer (GC) is the 5th most common cancer and the 3rd leading cause of cancer mortality worldwide [1-4], with non-cardia gastric cancer (NCGC) representing over $80 \%$ of all GC. There are clear global variations with respect to burden of disease, with areas of high incidence (e.g., East Asia, South/ Central America) and comparatively lower incidence (e.g., USA) [1]. There is growing concern that the incidence and 
mortality rates are increasing among certain groups in the USA, including minorities [5-7] and women below age 50 years [7]. However, because screening does not routinely occur in the USA even among high risk groups, NCGC is most often diagnosed in the advanced stages when curative resection is no longer an option. Prognosis is poor ( $<30 \% 5$-year survival), since effective therapy for unresectable GC currently does not exist. By contrast, if NCGC is diagnosed at a resectable stage, 5-year overall survival is significantly higher and approaches $95-99 \%$ for early gastric cancer (EGC) — defined as NCGC that invades no deeper than the submucosa $[8,9]$.

NCGC is believed to arise from the stepwise progression of chronic gastritis to atrophic gastritis (AG), intestinal metaplasia (IM), and dysplasia before malignant transformation to gastric adenocarcinoma [10,11]. Infection with Helicobacter pylori $(H$. pylori) is thought to be the primary trigger for these mucosal changes. Analogous to colorectal carcinogenesis, the detectable stepwise progression in gastric mucosa that takes place over many years, coupled with established safe endoscopic interventions for early stage disease, provides opportunity for screening, surveillance, and potentially curative therapeutic management and mortality benefit. Due to the high burden of disease and this understanding of the natural disease course, years ago Japan and South Korea (hereafter Korea) implemented national screening protocols, which have now corresponded to a reduction in overall GC-related mortality by at least $50-60 \%$, attributed to earlier detection and curative resection [12-15]. Other countries do not routinely screen for NCGC. While the reasons for this are many, they include issues such as insufficient economic and healthcare infrastructure, lack of awareness of high-risk groups on the part of healthcare providers [16], and patient-related factors. We previously reported that providers working with multiethnic populations in New York City (NYC) often did not recognize the higher burden of NCGC among certain racial and ethnic groups, including Asian-Americans, Blacks, Hispanics, and immigrant groups from endemic areas like Russia and Eastern Europe [2,16-18]. This occurred despite the fact that NCGC incidence rates in these groups are estimated to be at least two to three times higher than the USA-born White population [18-21]. Notably, the incidence of NCGC in Korean-Americans is similar to colorectal cancer in the USA population-a cancer routinely screened for-and is estimated to be over 5 times higher than the incidence of NCGC among USA-born Whites [18]. In fact, it has already been shown that offering selected GC screening for high-risk races and ethnicities is a highly cost-effective intervention in the USA [22]. Cost-effectiveness, however, does not necessarily equate to clinical effectiveness. While it stands to reason that the cost benefit for selected screening and surveillance is related to the identification of resectable NCGC, this has not been previously demonstrated in an at-risk USA population.

The primary aim of our study, therefore, was to determine whether having an upper endoscopy that was negative for malignancy (analogous to a screening endoscopy) prior to the index upper endoscopy that diagnosed NCGC was associated with a higher likelihood of curable stage NCGC at diagnosis compared to individuals who did not have an upper endoscopy prior to their index diagnosis. We secondarily aimed to identify other risk factors associated with curable stage NCGC at diagnosis.

\section{METHODS}

\section{Study Population}

This retrospective cross-sectional study was approved by the Institutional Review Board at Mount Sinai Hospital. Patients were identified via a query of a comprehensive pathology database, which includes endoscopic biopsies and resected specimens for cases of GC diagnosed by endoscopy and reviewed by the institution's expert gastrointestinal (GI) pathologists between January 2008 and May 2016. Each medical record was reviewed to confirm the histologic diagnosis of primary gastric adenocarcinoma and to ensure the availability of the endoscopic and histologic findings. Inclusion criteria were confirmed histologic diagnosis of gastric adenocarcinoma, confirmed location of cancer distal to the gastric cardia (i.e., at least $5 \mathrm{~cm}$ distal to the gastroesophageal junction), no prior documented history of GC of any type, and documentation of at least one endoscopy, which could have been the endoscopy during which NCGC was diagnosed (hereafter index endoscopy) or an endoscopy prior to their index endoscopy during which no malignancy was diagnosed (hereafter negative endoscopy). Patients were excluded if they had a diagnosis of primary adenocarcinoma of the gastric cardia or gastroesophageal junction, if they had a prior diagnosis of any GC, if they had any prior gastric surgeries, or if there was not documentation of at least one endoscopy. 


\section{Data Collection}

Patient demographics and baseline information including age, sex, race/ethnicity, smoking status, alcohol use, and family history of GC were recorded. We recorded the clinical presentation and indication for the index endoscopy during which NCGC was diagnosed. We documented any history of a negative endoscopy prior to the index endoscopy, as well as the respective time interval between the negative endoscopy and the index endoscopy diagnosing NCGC. Full endoscopic reports from endoscopies performed at outside institutions were included. All pathology obtained from an outside institution was re-reviewed by one of our institution's expert Gl pathologist and served as the final pathologic diagnosis. Background histologic findings at the time of the index NCGC diagnosis were documented as to the presence of AG, IM, and dysplasia (low-grade [LGD] and/or high-grade [HGD]). Pre-neoplasia was categorized as "multifocal" if documented in both the antrum and corpus. The presence or absence of active $H$. pylori infection was determined based on immunostain. For patients who were not actively infected with $H$. pylori based

Table 1. Demographics and clinical characteristics

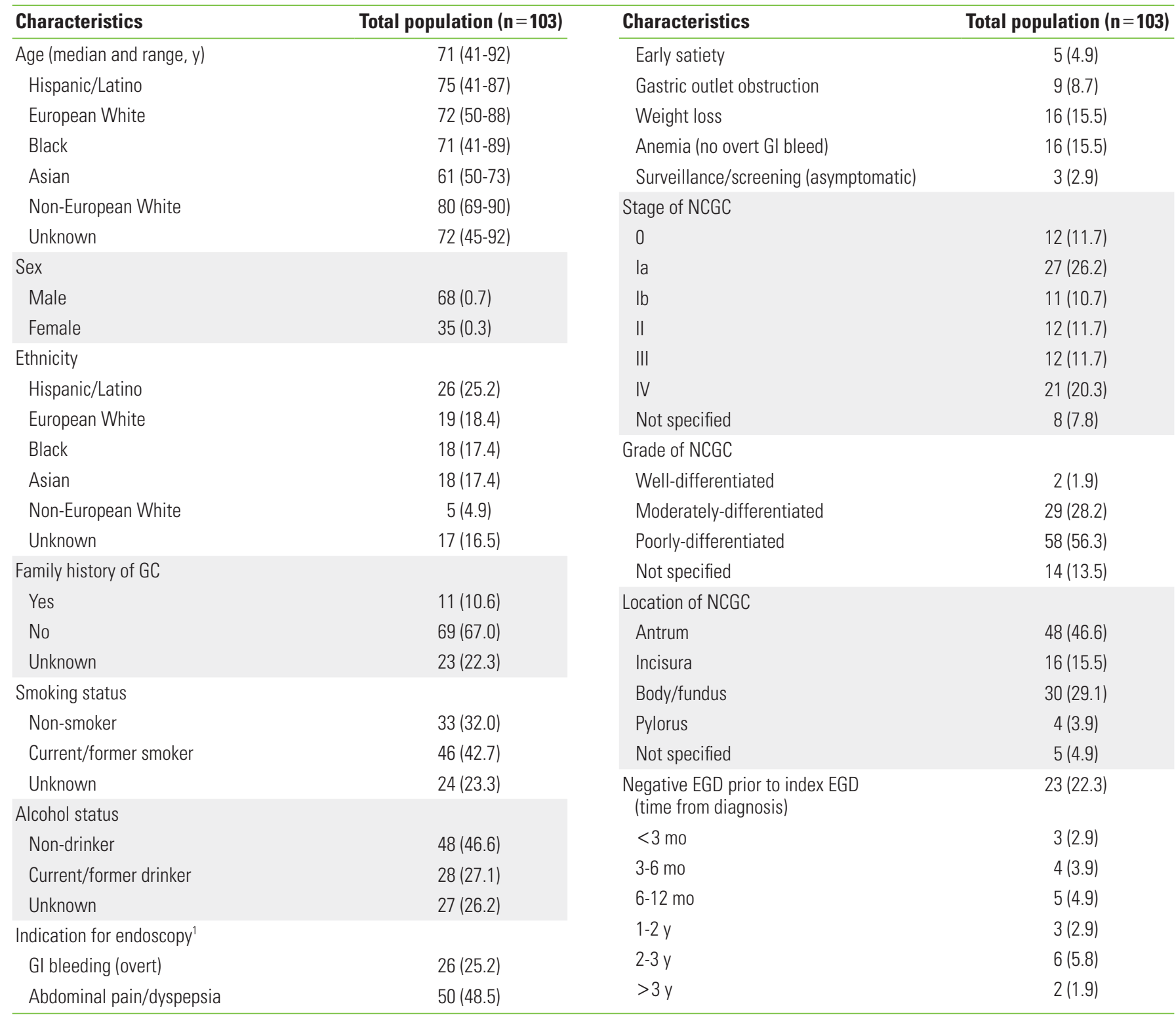

Values are presented as number (\%).

GC, gastric cancer; NCGC, non-cardia gastric cancer; GI, gastrointestinal; EGD, esophagogastroduodenoscopy.

${ }^{1}$ Indication for the index endoscopy on which NCGC was diagnosed was known in $91.2 \%$ of patients $(n=94 / 103)$. There was often more than one indication for endoscopy. 
on histologic immunostain, the chart was reviewed for evidence of prior positive serology or evidence of prior treatment. Cancer grade and stage at time of diagnosis were documented. Anatomic/prognostic stages were determined according to the American Joint Committee on Cancer, 7th edition [23]. Stage 0 and la tumors were classified as "EGCs" (curable stage disease) for this study. We did not include stage $\mathrm{lb}$ in this definition since stage $\mathrm{lb}$ includes both $\mathrm{T} 1 \mathrm{~N} 1$ and $\mathrm{T} 2 \mathrm{~N} 0$ and the latter are not amenable to modern endoscopic resection techniques due to invasion deeper than the submucosa.

\section{Outcomes}

The primary outcome was the frequency of curable stage NCGC at diagnosis in patients with an endoscopy negative for malignancy prior to their index endoscopy that diagnosed NCGC, compared to patients without a negative endoscopy prior to their index endoscopy. Secondary analyses included determination of factors associated with curable stage NCGC at diagnosis.

\section{Statistical Analysis}

Descriptive, univariate, and multivariate analyses were carried out using Stata version 11.0 (StataCorp., College Station, TX, USA). Logistic regression was used to identify factors associated with curable stage NCGC at diagnosis. In addition to clinically relevant covariates determined a priori, parameters with $p$-value $<0.30$ on univariate analyses were included in the multivariate models. Statistical significance was determined at the $p$-value $<0.05$ level.

\section{RESULTS}

\section{Demographics and Clinical Characteristics}

A total of 103 patients (70\% male) met criteria for inclusion (Table 1). The median age at the time of NCGC diagnosis was 71 (range, 41-92) years. Of the overall cohort, 25.2\% were Hispanic/Latino, 18.4\% European White, 17.4\% Asian, and 17.4\% Black, while $4.9 \%$ of the cohort was non-European White. Ap-

Table 2. Demographic and clinical characteristics based on EGD performed prior to index NCGC diagnosis

\begin{tabular}{|c|c|c|c|c|c|c|c|}
\hline Characteristic & $\begin{array}{c}\text { Prior EGD } \\
(\mathbf{n}=\mathbf{2 3})\end{array}$ & $\begin{array}{l}\text { No prior EGD } \\
(n=70)\end{array}$ & $p$-value & Characteristic & $\begin{array}{c}\text { Prior EGD } \\
\quad(n=23)\end{array}$ & $\begin{array}{l}\text { No prior EGD } \\
(n=70)\end{array}$ & $p$-value \\
\hline Age (median and range, y) & $70(51-92)$ & $71(41-90)$ & 0.89 & Abdominal pain/dyspepsia & $11(47.8)$ & $36(51.4)$ & 0.71 \\
\hline Sex & & & 0.22 & Early satiety & $2(8.7)$ & $3(4.3)$ & 0.30 \\
\hline Male & $14(60.9)$ & $52(74.3)$ & & Gastric outlet obstruction & $1(4.3)$ & $8(11.4)$ & 0.68 \\
\hline Female & $9(39.1)$ & $18(25.7)$ & & Weight loss & $3(13.0)$ & $13(18.6)$ & 0.74 \\
\hline Ethnicity & & & 0.31 & Anemia (no overt Gl bleed) & $4(17.4)$ & $11(15.7)$ & 0.73 \\
\hline Hispanic/Latino & $6(26.1)$ & $21(30.0)$ & 0.58 & NCGC stage & & & 0.001 \\
\hline European White & $8(34.8)$ & $11(15.7)$ & 0.06 & 0 & $8(34.8)$ & $3(4.3)$ & \\
\hline Black & $3(13.0)$ & $17(24.2)$ & 0.29 & la & $8(34.8)$ & $17(24.3)$ & \\
\hline Asian & $6(26.1)$ & $15(21.4)$ & 0.41 & $\mathrm{lb}$ & $1(4.3)$ & $8(11.4)$ & \\
\hline Non-European White & $0(0.0)$ & $6(8.6)$ & 0.57 & $\|$ & $2(8.7)$ & $7(10.0)$ & \\
\hline Family history of GC & & & 0.44 & III & $0(0.0)$ & $12(17.1)$ & \\
\hline Yes & $4(17.4)$ & $7(10.0)$ & & IV & $3(13.0)$ & $18(25.7)$ & \\
\hline No & $12(52.2)$ & $44(62.9)$ & & Not specified & $1(4.3)$ & $5(7.1)$ & \\
\hline Not specified & $7(30.4)$ & $19(27.1)$ & & NCGC grade & & & 0.27 \\
\hline Smoking status & & & 0.12 & Well-differentiated & $1(4.3)$ & $1(1.5)$ & \\
\hline Never & $11(47.8)$ & $19(27.1)$ & & Moderately-differentiated & $3(13.0)$ & $23(35.4)$ & \\
\hline Current/former & $9(39.1)$ & $35(50.0)$ & & Poorly-differentiated & $10(43.4)$ & $41(63.8)$ & \\
\hline Not specified & $3(13.0)$ & $16(22.9)$ & & Not specified & $9(39.1)$ & $5(7.1)$ & \\
\hline Alcohol status & & & 0.18 & NCGC location & & & 0.89 \\
\hline Never & $15(65.2)$ & $30(42.9)$ & & Antrum & $11(47.8)$ & $32(45.7)$ & \\
\hline Current/former & $5(21.7)$ & $23(32.9)$ & & Incisura & $3(13.0)$ & $12(17.1)$ & \\
\hline Not specified & $3(13.0)$ & $17(24.3)$ & & Body/fundus & $7(30.4)$ & $22(31.4)$ & \\
\hline Indication for endoscopy & & & & Pylorus & $1(4.4)$ & $3(4.3)$ & \\
\hline GI bleeding (overt) & $4(17.4)$ & $20(28.6)$ & 0.57 & Not specified & $1(4.4)$ & $1(1.4)$ & \\
\hline
\end{tabular}

Values are presented as number (\%).

EGD, esophagogastroduodenoscopy; NCGC, non-cardia gastric cancer; GC, gastric cancer; GI, gastrointestinal.

'Detailed information about prior EGD was unavailable for 10 patients (excluded from prior analysis). 
proximately $43 \%$ were current or former smokers, and $27.1 \%$ were current or former alcohol drinkers. The indication for the index endoscopy on which the NCGC was diagnosed was available in 94 patients (91.2\%). Since endoscopic screening of asymptomatic individuals is not routine in the USA, the majority of patients were symptomatic at the time of NCGC diagnosis, with less than $3 \%$ of patients $(n=3)$ having "surveillance" as their documented indication for endoscopy. Most patients had one or more of the following symptoms at presentation: abdominal pain/dyspepsia, Gl bleed (overt or suspected occult), weight loss, gastric outlet obstruction, or early satiety (Table 1).

Practically all (98\%) patients had histological evidence of background pre-neoplasia at the time of NCGC diagnosis (data not shown). One-third had AG (34\%), while all 101 patients with pre-neoplasia had some form of IM. Only $10 \%$ of patients had active $H$. pylori infection by immunostain. No additional patients were identified as formerly positive based on available H. pylori serology or based on documentation of prior anti-H. pylori therapy.

Over half of patients had poorly-differentiated adenocarcinoma (56.3\%), with only $1.9 \%$ of the cohort having well-differentiated histology. Antral location was most common (46.6\%). At diagnosis, $37.9 \%$ of patients had curable stage NCGC (11.7\% stage $0,26.2 \%$ stage la), while over $20 \%$ had metastatic disease. Among patients with stage III or IV NCGC at presentation, $90.9 \%$ had not had an endoscopy prior to their diagnosis.

\section{Primary Outcome}

Over $22 \%(n=23)$ had at least one negative endoscopy prior to their index endoscopy that diagnosed NCGC, while $68 \%$ $(n=70)$ had no endoscopy prior to their index endoscopy diagnosing NCGC (Table 2). There was no difference in age, sex, race/ethnicity, family history of GC, smoking status, alcohol status, or indication for endoscopy between those who had an endoscopy and those who did not have an endoscopy prior to their index endoscopy diagnosing NCGC. There was also no difference in the location or histologic grade of NCGC. Notably, however, nearly $69.6 \%$ of the NCGCs diagnosed in patients with a prior negative endoscopy were stage 0 or la (curable stages) compared to only $28.6 \%$ in patients who did not have a prior endoscopy $(p=0.001)$. Similarly, only $13.0 \%$ of NCGCs diagnosed in the patients who had had a prior negative endoscopy were stage III or IV, compared to nearly $42.8 \%$ among patients who had not had a prior endoscopy $(p=0.001$ ) (Table 2). The median time from prior negative endoscopy to the endoscopy with the index NCGC diagnosis was 343 days.

Table 3. Univariate and multivariate analysis of associations with NCGC stage $>1$ a at diagnosis ${ }^{1}$

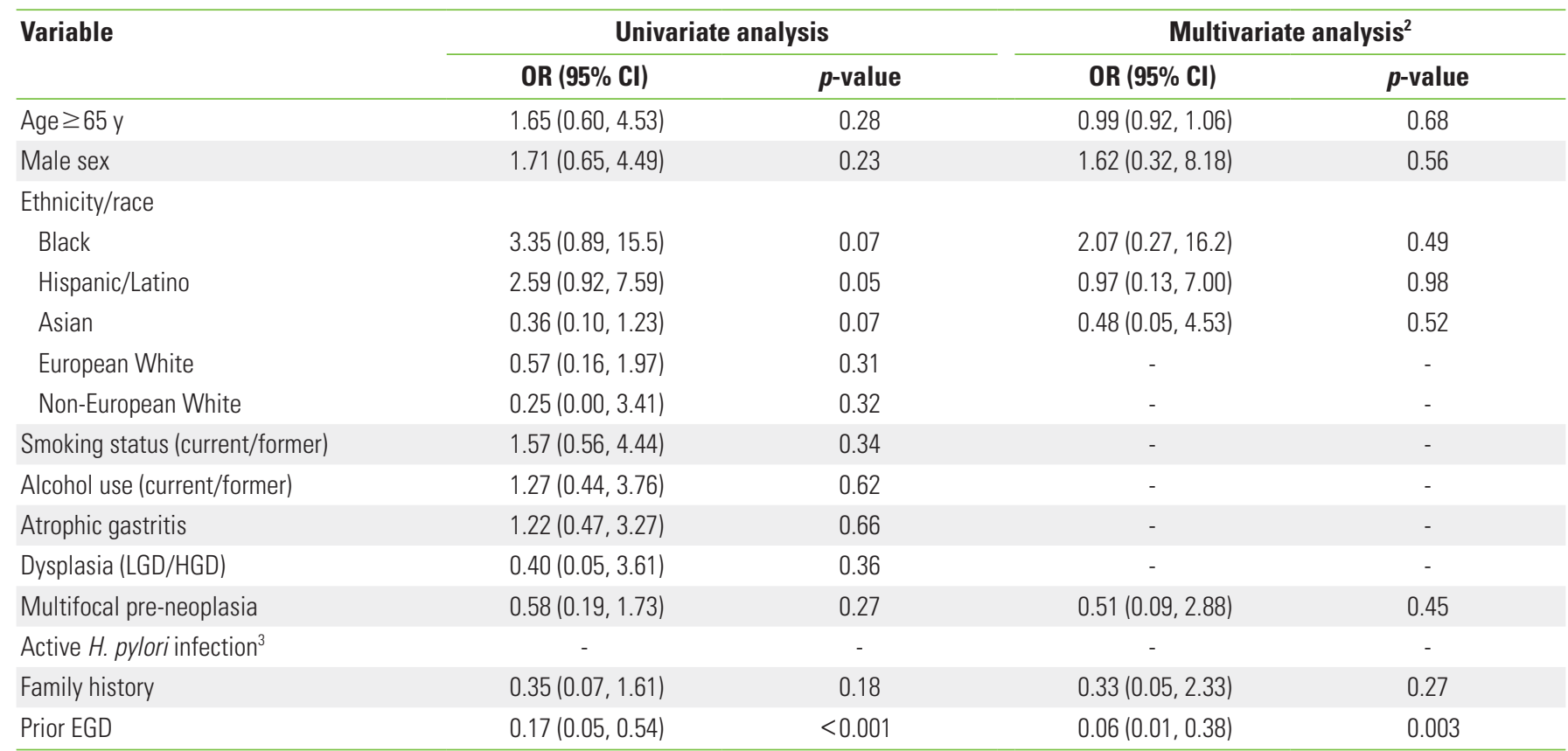

NCGC, non-cardia gastric cancer; OR, odds ratio; Cl, confidence interval; LGD, low-grade dysplasia; HGD, high-grade dysplasia; EGD, esophagogastroduodenoscopy. 'Univariate and multivariate analysis for intestinal metaplasia could not be performed given its nearly universal presence in the cohort.

${ }^{2}$ Only factors with $p<0.3$ in the univariate analysis were included in multivariable analysis.

${ }^{3}$ No patients with stage 0 or 1a NCGC had active H. pylori infection, precluding univariate and multivariate analyses. 


\section{Factors Associated With Non-cardia Gastric}

\section{Cancer Stage $>1$ a at Diagnosis}

On univariate analysis, having an upper endoscopy in the three years prior to the index endoscopy (compared to having no prior endoscopy) was strongly protective against NCGC stage $>$ la at diagnosis $(p<0.001)$. Being Asian was associated with a trend toward higher likelihood of being diagnosed with EGC compared to non-Asian groups ( $p=0.07$ ). By contrast, being Black or Hispanic/Latino was associated with greater odds of having NCGC stage $>$ la at diagnosis, although only the latter reached statistical significance $(p=0.07$ and $p=0.05$, respectively). No other factors were significantly associated with worse stage of NCGC at diagnosis. Having a negative endoscopy prior to the index diagnosis remained significantly protective on multivariable analysis and was associated with a $94 \%$ higher likelihood of having curable stage 0 or la NCGC at diagnosis $(p=0.003)$ (Table 3 ).

\section{DISCUSSION}

In this study of over 100 people diagnosed with NCGC at an urban tertiary referral center in NYC, we report a marked racial and ethnic minority predominance, with over $95 \%$ of those diagnosed with NCGC being Black, Hispanic/Latino, Asian, or Eastern European/Russian, and therefore constituting a highrisk group based on race and ethnicity alone $[2,17,18]$. Our findings support current literature that, while under-recognized, certain racial and ethnic groups bear a significantly higher burden of NCGC in the USA compared to USA-born Whites. Acknowledging the retrospective nature of our study, having an endoscopy within three years prior to the index NCGC diagnosis was the only factor significantly associated with lower stage NCGC at diagnosis (specifically EGC) in our cohort, which was notably high-risk nearly universally based on race and ethnicity alone. Our findings warrant confirmation with larger studies that specifically include a lower-risk cohort diagnosed with NCGC as comparison.

Indeed, $70 \%$ of patients who had a negative endoscopy prior to their index endoscopy had their cancers diagnosed at a stage that is historically associated with $95-99 \%$ 5-year survival when adequately resected, either endoscopically or surgically [24-28]. Our findings are in line with studies from highrisk populations, namely East Asia, showing that screening or surveillance endoscopy is associated with lower stage disease at diagnosis $[29,30]$. While a randomized controlled trial of en- doscopic screening compared to no screening would be the most robust approach to assess the impact of a screening and surveillance protocol, this would require a large populationbased cohort and is logistically not feasible in the USA given the overall low prevalence of NCGC. Moreover, the duration of follow-up needed to show mortality benefit would be quite long. Thus, there are no randomized controlled trials assessing the impact of NCGC screening even in high-prevalence countries such as Japan and Korea, which have established national screening protocols. Arguably, such trials would now be unethical since screening with upper endoscopy has been associated with at least a 30-65\% reduction in GC-related mortality in Japan $[12,14,31]$, and nearly $80 \%$ reduction in Japanese male age 40-75 [31], which is attributed to a marked increase in the percentage of NCGC diagnosed as EGCs. EGCs now represent almost $60 \%$ of the diagnosed NCGCs in Japan, compared to $15 \%$ prior to implementation of GC screening [4,32], which mirrors our data that nearly $70 \%$ of NCGC stage la or lower in our cohort were diagnosed in patients who had a negative endoscopy prior to their index endoscopy diagnosing NCGC. A prospective study from the UK, another overall low prevalence area, showed that patients with pre-neoplasia who were enrolled in an endoscopic GC surveillance program-i.e., a high-risk group in an otherwise relatively low prevalence area-had earlier stage GC (defined as stage I or II) at diagnosis $(p<0.05)$ [33]. To our knowledge, ours is the first study suggesting a possible clinical benefit of endoscopy for NCGC screening for high-risk subgroups based on racial and ethnic background in a country with otherwise low NCGC prevalence. Reflective of this, a guideline statement by the Standards of Practice Committee of the American Society for Gastrointestinal Endoscopy (ASGE) recommends considering screening new USA immigrants above the age of 40 from high-risk endemic regions (Japan, Korea, China, Russia, and South America) for GC with upper endoscopy, particularly in those with first-degree relatives with a history of GC [34]. Notably, no recommendations are offered for other high-risk groups in the USA, including later-generation immigrants from endemic areas, Hispanic/Latinos and Black Americans, despite an acknowledgement by the ASGE that these groups also have a significantly higher incidence of GC compared to USA-born Whites. While we acknowledge that our population is technically not a screening population because the majority had symptoms listed as the endoscopic indication, publications from the cancer surveillance programs in high preva- 
lence countries like Korea also acknowledge their population is likely not a "true" screening population, since it is not possible to exclude symptomatic individuals [30].

The observation that NCGC risk is higher among certain racial/ethnic groups in the USA more likely reflects cultural, dietary, socioeconomic, and other social determinants of health, rather than an intrinsic predisposition to GC [35]. This is supported by migration data, which suggest that after approximately 2-3 generations, immigrants from areas endemic for NCGC display the prevalence of their host country, a phenomenon that is attributed to dietary acculturation, better sanitation, and possibly lower H. pylori prevalence [36]. Our finding of nearly universal background pre-neoplasia, specifically IM, suggests the step-wise progression of preneoplasia prior to final malignant transformation occurs irrespective of race/ethnicity. Despite H. pylori's purported role as the primary trigger for the pre-neoplastic cascade, only a small percentage of our cohort had active infection; this suggests not only a limited role for $\mathrm{H}$. pylori testing in identifying individuals who may benefit from screening and surveillance to detect early stage NCGC, but it also suggests that while $H$. pylori likely underlies the initial carcinogenesis cascade, other factors contribute to the progression of preneoplasia to neoplasia.

Strengths of our study include a highly diverse patient population with access to detailed medical records and accurate staging of NCGC, as well as expert pathology review of all biopsy specimens. Important limitations of our study include the cross-sectional and retrospective nature, as well as the relatively small cohort size limiting our power to detect differences, particularly among racial and ethnic groups. Our study is from a single, tertiary center and may limit generalizability. While we included endoscopies performed at outside facilities if performed prior to the index endoscopy diagnosing NCGC, outside endoscopies performed but not documented in the medical record would be missed. However, this would be considered non-differential misclassification and tend to bias towards the null. Given the magnitude of our effect estimate, it is unlikely that these minimal missing data would be sufficient to nullify the protective effect afforded by a prior negative endoscopy reported here. As previously noted, although our cohort is possibly representative of the population in the USA who would benefit from GC screening and surveillance based on published prevalence and cost-effectiveness data $[20,22$, 37-40], our ability to draw strong conclusions regarding factors associated with early stage GC at diagnosis is limited by the fact that our cohort is almost universally high-risk. Although only $5 \%$ of patients in our cohort were non-European Whites and limited parallel analysis of a theoretically lowerrisk group for NCGC, this critically highlights the disparity of disease incidence in the USA and the need for increased attention to the differential disease burden in these groups.

In conclusion, in a racially and ethnically diverse USA population, we found that having an upper endoscopy that was negative for malignancy prior to the index endoscopy diagnosing NCGC was associated with a significantly higher likelihood of having curable stage disease at presentation compared to patients who did not have an endoscopy prior to their index diagnosis. Targeted endoscopic screening and surveillance for select groups deemed higher-risk might positively impact GC-related outcomes by detecting disease at an earlier, potentially curable, stage. We hope that the findings of the present study will both stimulate and inform future studies validating risk-stratification models for NCGC screening and surveillance in the USA.

\section{CONFLICT OF INTEREST}

The authors have no conflicts of interest associated with the material presented in this paper.

\section{ACKNOWLEDGEMENTS}

This study was partly funded by a grant from the AHRQ awarded to SCS (K12 HS026395-01).

\section{ORCID}

Shailja C. Shah https://orcid.org/0000-0002-2049-9959

Alexandros D. Polydorides https://orcid.org/0000-00018604-0756

\section{REFERENCES}

1. Bray F, Ren JS, Masuyer E, Ferlay J. Global estimates of cancer prevalence for 27 sites in the adult population in 2008. Int J Cancer 2013;132(5):1133-1145.

2. Bertuccio P, Chatenoud L, Levi F, Praud D, Ferlay J, Negri E, et al. Recent patterns in gastric cancer: a global overview. Int J Cancer 2009;125(3):666-673.

3. Sigon R, Canzonieri V, Rossi C. Early gastric cancer: a single- 
institution experience on 60 cases. Suppl Tumori 2003;2(5): S23-S26.

4. Shimizu S, Tada M, Kawai K. Early gastric cancer: its surveillance and natural course. Endoscopy 1995;27(1):27-31.

5. Siegel RL, Fedewa SA, Miller KD, Goding-Sauer A, Pinheiro PS, Martinez-Tyson D, et al. Cancer statistics for Hispanics/Latinos, 2015. CA Cancer J Clin 2015;65(6):457-480.

6. Merchant SJ, Kim J, Choi AH, Sun V, Chao J, Nelson R. A rising trend in the incidence of advanced gastric cancer in young Hispanic men. Gastric Cancer 2017;20(2):226-234.

7. Anderson WF, Rabkin CS, Turner N, Fraumeni JF Jr, Rosenberg PS, Camargo MC. The changing face of noncardia gastric cancer incidence among US non-Hispanic whites. J Natl Cancer Inst 2018;110(6):608-615.

8. Alfaro EE, Lauwers GY. Early gastric neoplasia: diagnosis and implications. Adv Anat Pathol 2011;18(4):268-280.

9. Gotoda T. Endoscopic resection of early gastric cancer: the Japanese perspective. Curr Opin Gastroenterol 2006;22(5): 561-569.

10. Sipponen P, Kimura K. Intestinal metaplasia, atrophic gastritis and stomach cancer: trends over time. Eur J Gastroenterol Hepatol 1994;6 Suppl 1:S79-S83.

11. Kuipers EJ, Uyterlinde AM, Peña AS, Roosendaal R, Pals G, Nelis $\mathrm{GF}$, et al. Long-term sequelae of Helicobacter pylori gastritis. Lancet 1995;345(8964):1525-1528.

12. Hamashima C. Current issues and future perspectives of gastric cancer screening. World J Gastroenterol 2014;20(38):1376713774.

13. Hamashima C, Shibuya D, Yamazaki H, Inoue K, Fukao A, Saito $\mathrm{H}$, et al. The Japanese guidelines for gastric cancer screening. Jpn J Clin Oncol 2008;38(4):259-267.

14. Hamashima C, Ogoshi K, Narisawa R, Kishi T, Kato T, Fujita K, et al. Impact of endoscopic screening on mortality reduction from gastric cancer. World J Gastroenterol 2015;21(8):24602466.

15. Matsumoto S, Yoshida Y. Efficacy of endoscopic screening in an isolated island: a case-control study. Indian J Gastroenterol 2014;33(1):46-49.

16. Shah SC, Itzkowitz SH, Jandorf L. Knowledge gaps among physicians caring for multiethnic populations at increased gastric cancer risk. Gut Liver 2018;12(1):38-45.

17. Espey DK, Wu XC, Swan J, Wiggins C, Jim MA, Ward E, et al. Annual report to the nation on the status of cancer, 1975-2004, featuring cancer in American Indians and Alaska Natives. Cancer 2007;110(10):2119-2152.
18. Kim Y, Park J, Nam BH, Ki M. Stomach cancer incidence rates among Americans, Asian Americans and Native Asians from 1988 to 2011. Epidemiol Health 2015;37:e2015006.

19. Fennerty MB, Emerson JC, Sampliner RE, McGee DL, Hixson LJ, Garewal HS. Gastric intestinal metaplasia in ethnic groups in the southwestern United States. Cancer Epidemiol Biomarkers Prev 1992;1(4):293-296.

20. Miller BA, Chu KC, Hankey BF, Ries LA. Cancer incidence and mortality patterns among specific Asian and Pacific Islander populations in the U.S. Cancer Causes Control 2008;19(3):227256.

21. Wu X, Chen VW, Andrews PA, Ruiz B, Correa P. Incidence of esophageal and gastric cancers among Hispanics, non-Hispanic whites and non-Hispanic blacks in the United States: subsite and histology differences. Cancer Causes Control 2007;18(6): 585-593.

22. Saumoy M, Schneider Y, Shen N, Kahaleh M, Sharaiha RZ, Shah SC. Cost effectiveness of gastric cancer screening according to race and ethnicity. Gastroenterology 2018;155(3):648-660.

23. Washington K. 7th edition of the AJCC cancer staging manual: stomach. Ann Surg Oncol 2010;17(12):3077-3079.

24. Okada K, Fujisaki J, Yoshida T, Ishikawa H, Suganuma T, Kasuga A, et al. Long-term outcomes of endoscopic submucosal dissection for undifferentiated-type early gastric cancer. Endoscopy 2012;44(2):122-127.

25. Yasuda K, Shiraishi N, Suematsu T, Yamaguchi K, Adachi Y, Kitano $S$. Rate of detection of lymph node metastasis is correlated with the depth of submucosal invasion in early stage gastric carcinoma. Cancer 1999;85(10):2119-2123.

26. Wang S, Zhang Z, Liu M, Li S, Jiang C. Endoscopic resection compared with gastrectomy to treat early gastric cancer: a systematic review and meta-analysis. PLoS One 2015;10(12): e0144774.

27. Choi IJ, Lee JH, Kim YI, Kim CG, Cho SJ, Lee JY, et al. Long-term outcome comparison of endoscopic resection and surgery in early gastric cancer meeting the absolute indication for endoscopic resection. Gastrointest Endosc 2015;81(2):333-341.

28. Pyo JH, Lee H, Min BH, Lee JH, Choi MG, Lee JH, et al. Longterm outcome of endoscopic resection vs. surgery for early gastric cancer: a non-inferiority-matched cohort study. Am J Gastroenterol 2016;111(2):240-249.

29. Khanderia E, Markar SR, Acharya A, Kim Y, Kim YW, Hanna GB. The influence of gastric cancer screening on the stage at diagnosis and survival: a meta-analysis of comparative studies in the Far East. J Clin Gastroenterol 2016;50(3):190-197. 
30. Choi KS, Jun JK, Suh M, Park B, Noh DK, Song SH, et al. Effect of endoscopy screening on stage at gastric cancer diagnosis: results of the National Cancer Screening Programme in Korea. Br J Cancer 2015;112(3):608-612.

31. Hosokawa O, Miyanaga T, Kaizaki Y, Hattori M, Dohden K, Ohta $\mathrm{K}$, et al. Decreased death from gastric cancer by endoscopic screening: association with a population-based cancer registry. Scand J Gastroenterol 2008;43(9):1112-1115.

32. Noguchi Y, Yoshikawa T, Tsuburaya A, Motohashi H, Karpeh MS, Brennan MF. Is gastric carcinoma different between Japan and the United States? Cancer 2000;89(11):2237-2246.

33. Whiting JL, Sigurdsson A, Rowlands DC, Hallissey MT, Fielding JW. The long term results of endoscopic surveillance of premalignant gastric lesions. Gut 2002;50(3):378-381.

34. Cash BD, Banerjee S, Anderson MA, Ben-Menachem T, Decker $\mathrm{GA}$, Fanelli RD, et al. Ethnic issues in endoscopy. Gastrointest Endosc 2010;71(7):1108-1112.

35. Karimi P, Islami F, Anandasabapathy S, Freedman ND, Kamangar F. Gastric cancer: descriptive epidemiology, risk factors, screening, and prevention. Cancer Epidemiol Biomarkers Prev 2014;23(5):700-713.

36. Maskarinec G, Noh JJ. The effect of migration on cancer incidence among Japanese in Hawaii. Ethn Dis 2004;14(3):431439.

37. New York State. Stomach cancer incidence and mortality by age group, New York State Excl New York City, 2009-2013 [cited 2018 Mar 12]. Available from: https://www.health.ny.gov/ statistics/cancer/registry/table6/tb6stomachupstate.htm.

38. Lui FH, Tuan B, Swenson SL, Wong RJ. Ethnic disparities in gastric cancer incidence and survival in the USA: an updated analysis of 1992-2009 SEER data. Dig Dis Sci 2014;59(12):3027-3034.

39. Lee J, Demissie K, Lu SE, Rhoads GG. Cancer incidence among Korean-American immigrants in the United States and native Koreans in South Korea. Cancer Control 2007;14(1):78-85.

40. National Cancer Institute. Surveillance, Epidemiology, and End Results (SEER) program [cited 2018 Mar 13]. Available from: http://seer.cancer.gov. 